\title{
Formation and Evolution of Galaxies and Black Holes
}

\author{
Cui-xiang Zhong \\ Department of Science and Technology, Jiangxi Normal University, Nanchang 330022, China
}

\begin{abstract}
In the past, people did not realize the formation and structure of galaxies. They even mistook the black holes hidden in the center of a galaxy as independent celestial objects, making black holes mysterious and unbelievable. It was only after the author studied and discovered the laws of the formation and evolution of satellites, planets and stars that he put forward the scientific theory of galaxy formation and evolution, therefore revealing the hierarchical structure of galaxy and the existence and characteristics of black holes as the main nodes of galactic structure.
\end{abstract}

Key words: Galactic structure, galaxy formation, galaxy evolution, black holes, black hole area.

\section{Introduction}

As early as 1930 s, it has been recognized that galaxies in the universe are not randomly distributed, but because of the lack of two dimensional observational data, there has been no breakthrough in the understanding of large-scale structures. Only until 1980s, with the continuous deepening of red-shift survey, could one clearly describe the three-dimensional distribution of galaxies in large scale structure, and found that galaxies have lumpy structures. But the structural relationship among stars is still unclear, even the existence of black holes in galaxies is suspected.

In fact, the proposition of the concept about black holes in galaxy can be traced back to British scholar John Michell and French astronomer P. Laplace at the end of the eighteenth century, in a sense they had foreseen the existence of black holes. In 1916, Albert Einstein published his theory of general relativity, having also predicted the existence of black holes. Modern experiments have also found evidence of black holes. For example, in 1970, the United States' satellite Liberty discovered the first black hole, then some super-massive black holes have also been found [1-3]. Chinese astronomers have also made great

Corresponding author: Cui-xiang Zhong, Ph.D., associate professor, research fields: astrophysics and computer applications. achievements in the research field of black holes. In 2005, the working group led by Zhiqiang Shen of Shanghai Astronomical Observatory of Chinese Academy of Sciences found some conclusive evidence supporting that there exists a super massive black holes in the center of Milky Way. In 2015, the international team led by astronomers from Peking University in China and from the University of Arizona announces their findings of a quasar, with its central black hole mass of 12 billion solar masses and the luminosity of 420 trillion suns is at a distance of 12.8 billion light-years from Earth [4]. However, in recent years, some internationally renowned experts have published papers saying there is no black hole in the universe. So, we can see that there is still controversy about the existence of black holes. But, according to the galaxy formation and evolution theory proposed by the author, it is easy to reveal the structure of galaxies and the formation and evolution of black holes.

\section{The Formation and Evolution of Galactic Structure}

\subsection{The Formation and Evolution of Planetary Systems}

According to the Solar System's formation law described in some papers of the author [4], a star can produce several planets around it, and each planet can also produce zero or several satellites around it, 
therefore forming a planetary system, which is a hierarchical structure [5].

In a hierarchical galaxy, any star other than root-star was originally generated as a satellite of its parent planet. These satellites unceasingly incorporated the nebula materials near the orbits to become larger and larger, and gradually moved away from its parent planet under the impact of moving objects or the effect of the centrifugal force produced by the accelerating rotation of its parent planet. Especially, when the parent planet becomes a fixed star, its satellites would become planets. During the planets' revolution around its parent star, they unceasingly incorporate the nebula materials near the orbits to become larger and larger and may also generate new satellites. So except some small planets (like Mercury and Venus of the Solar System) near the parent star, other larger planets have their own thick atmospheres. During the normal rotation of a planet (such as Earth) around its parent star (such as the Sun), the atmospheric pressure on the trailing hemisphere of the planet is higher than the other hemisphere, effectively increasing its speed, thus making the planet gradually move away from the parent-star along a spiral line $[5,6]$. As the planets gradually move away from their parent star, the gravitational attractions of the parent star to these planets also gradually become smaller, consequently these planets' self-rotation will gradually accelerate, making their satellites' revolution faster, eventually causing the satellites to move away from their planet. In addition, with the growth of the mass of a planet, the planet will shrinks from time to time, but conserving the angular momentum of the planet, so a decrease in the rotational inertia results in an increase in the rotation speed of the planet, and consequently also an increase in the revolution speed of the satellites, making the satellites move away from the planet.

\subsection{The Formation and Evolution of Stars}

(1) The birth of stars
During the rotation of a planet around its parent star, it unceasingly absorbed dust and gases near the orbits, making its mass increase gradually and its atmosphere become thicker and thicker. When a planet's mass becomes very large, its core temperature and gas density are high enough to initiate and maintain thermonuclear reactions, a new star is born [7].

(2) Young stars - main sequence star

After a star is born, it enters an evolutionary phase using hydrogen fusion as its major energy source. The outward expansion force of the gas inside the star and the inward contraction force induced by gravity are roughly balanced, making the star neither contract nor expand. So this is a relatively stable phase, the duration is about $90 \%$ of its whole life span, which is called the main sequence stage of a star, it is the prime of the star's life [7].

(3) Middle aged star — red giant star

During the rotation of a star around the center of galaxy, it unceasingly absorbed gases, dust and other interstellar matter near the orbits, the trapped material is barely able to escape from the besiege of the dense atmosphere of the star. Many light elements are fused into heavy elements by the thermonuclear reactions of the star. So when a star evolves to the end of its main sequence stage, its mass increases greatly and can reach several times the mass of the sun. The greater the mass of a star, the faster its energy is consumed, so it is increasingly difficult to capture external material to meet its energy consumption. Especially, old stars usually have complex sub-galaxies, including a series of planets, satellites and even young stars. These sub-galaxies move around the old star and compete for the resources originally belonging to the old star. When the hydrogen needed for the thermonuclear reactions in the core of the old star is insufficient, the thermonuclear reactions based on hydrogen fusion cannot proceed in the core of the old star. At this point the gravitational force is not balanced by the radiation pressure of the fusion, the core of the star will be compressed, and the temperature will rise sharply. 
After the temperature of the central helium ball is raised, the upper hydrogen-helium mixture clinging to the helium ball will be heated to the temperature of hydrogen fusion, therefore thermonuclear reaction will resume. So the helium ball gets bigger and bigger, and the hydrogen combustion layer expands outward correspondingly, making the astral outer material expand due to heating, thus the star be transformed to a red giant star $[8,9]$.

(4) Stars in later years-White dwarf

When a star evolves into its old age and a series of nuclear reactions of the star come to their end, the material in the star no longer undergoes fusion reactions, so the star has no source of energy. As a result, it cannot support itself by the heat generated by fusion against gravitational collapse, but is supported only by electron degeneracy pressure, causing it to be extremely dense. During the contraction of the star, a large amount of energy is released, making the white dwarf white hot, surface temperatures above $10,000{ }^{\circ} \mathrm{C}$. This is the reason for white dwarf white [10].

(5) Rise from the dead-Supernova

During the movement of a galaxy, a white dwarf not only unceasingly incorporates the nebula materials near the orbits to increase its own mass but also merges some impacting celestial bodies of any longer sub-galaxy, making a white dwarf evolve into a massive star. Undergoing a series of evolution, the star is transformed into an onion-like shell structure in which light elements are outside and heavy elements inside, and its core is mainly composed of iron. Because the mass of such a star is much greater than that of the earth, the thickness and density of cloud around the star is much greater than the thickness and density of thunder storm around the earth, so when such a star revolves around its parent star and whirls on its axis fast, lots of cloud clumps would bump each other to cause immense electricity and heat, finally leading supernova explosion. The luminosity of the supernova explosion may rise to several hundred million times as much as the sun's luminosity, making the star become a supernova [11].

(6) Late stages of stellar evolution-neutron star

During the evolution of a white dwarf into a massive star, supernova explosions may occur multiple times. During each explosion, there is an accompanying collapse, causing the material at the core to compress more tightly. When the mass of the white dwarf increase beyond the Chandra limit, which is 1.4 times the mass of the sun, especially when a huge celestial object hits it, a supernova explosion will occur immediately, and the gravitational attraction of the star core is strong enough to compress atomic nuclei and electrons into neutrons, transforming the white dwarf into a neutron star with a diameter of only a dozen kilometers and a density of one billion tons per cubic centimeter. As the star shrinks into a neutron star, its rotation speed increase greatly as a result of conservation of angular momentum, hence the newly formed neutron star rotates at up to several hundred times per second [12].

(7) The endpoints of star evolution-black hole

Neutron stars also have an upper limit to their mass, often referred to as the Oppenheimer-Volkov limit, which is about 3 times the Sun's mass. If the core of a star exceeds the Oppenheimer-Volkov limit at the end of its life, there is no force in any standard theory able to prevent it from collapsing completely into a small particle-black hole. There is a region around the particle, which exhibits such strong gravitational effects that nothing can escape from inside it [13].

\subsection{The Hierarchical Structure of Galaxy}

According to the Solar System's formation law described in some papers of the author: a star may have its own parent-star or higher ancestral stars, and can produce several planets around it, and each planet can also produce several satellites around it; after a satellite grows into a planet, it can also produce its next generation of satellites. Hence, the basic structure of a galaxy is a hierarchical structure composed of 
many generations of stars, like a tree, and the entire universe contains many such galaxies, like an endless forest [4]. In a typical galaxy structure, a path from the galaxy center as the root of the tree to one of the moons as the leaves of the tree may contain several black holes, several neutron stars, and several white dwarfs, 0 1 red giant stars, 1 main-sequence star, 1 planet and 1 satellite. Each branch of a galaxy usually has at most two layers of luminous stars, and these luminous stars are usually at the bottom of the galaxy [6].

Milky Way galaxy is just a complicated hierarchical structure composed of multiple generations of stars, and the Solar system is only a branch of it. Astronomical observation also shows that Milky Way galaxy has a huge disk structure, consisting of the center of the Milky Way and at least two spiral arms, which are 4,500 light years apart. Our sun is located at the Orion Arm of the Milky Way galaxy, 26,000 light years from the center of the Milky Way. Through a study of the star cluster disk of the Milky Way, astronomer Maria German found that the star cluster in the inner part of Milky Way is older, while the star cluster in the outer part is younger. Thus, it can be inferred that the formation of the Milky Way started from the inner part, then gradually evolved into a star cluster disk about 100,000 light years across. In the process of the Milky Way's growth, it has swallowed up a number of small galaxies, the celestial bodies from other galaxies had been merged into the interior of the Milky Way. So the sun is a fixed star generated from multiple generations of stars, Earth is a child-planet of the Sun, and the Moon is a last generation star. Similarly, the external galaxies observed by Hubble are also hierarchical structures, appearing long spiral arms. Astronomers have discovered a by far the largest supercluster of galaxies-Laniakea super-cluster, which consists of thousands of member galaxies including the Milky Way and other galaxies in the local group of galaxies as well as the nearby Virgo Cluster of galaxies, as is shown in Fig. 1, where green area is crowded with many galaxies marked by white points, white lines show how they center around the super-cluster, orange line marked the margin of Laniakea, and the blue point is the location of our earth.

In addition, for the same hierarchy, the higher the level of a star is, the longer its accumulation, therefore the greater it's mass. When the original star collapsed into a white dwarfs, neutron star or black hole, it conserved most of its angular momentum, but the new radius is only a tiny fraction of the original star's radius,

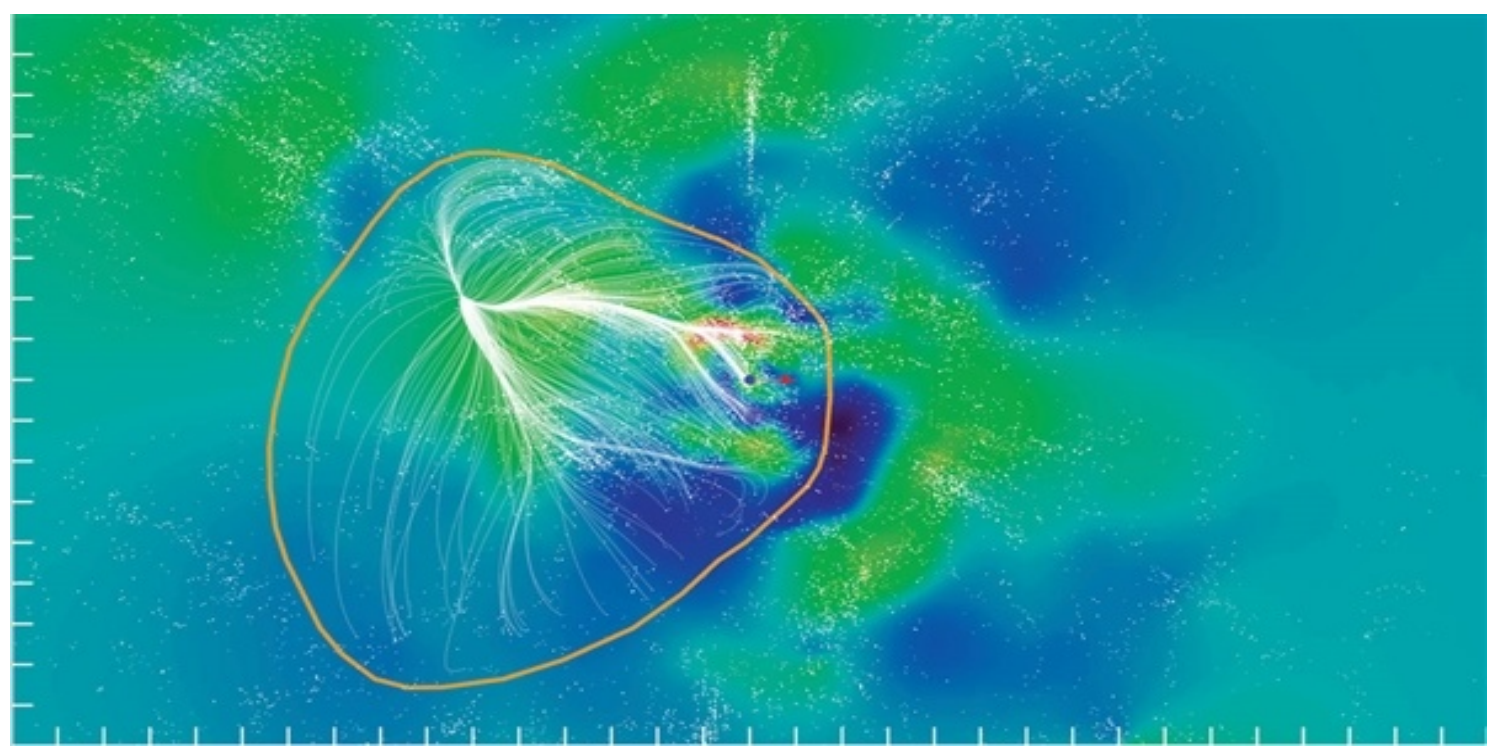

Fig. 1 Laniakea super-cluster with hierarchical structure. 
a decrease in the rotational inertia resulted in a rapid increase in the rotation speed, producing very high rotation rates. Hence, the higher the level of a star is, the greater is its rotation speed.

\section{The Formation and Evolution of Black Holes}

In fact, according to the galaxy formation theory described above, any galaxy is a hierarchical structure composed of many generations of stars and each generation consists of several brother stars. Such galaxy structure is not static, but in constant motion and change. A star of a later generation in a galaxy always revolves around its predecessors, i.e., a satellite moves around its parent planet, a planet moves around its parent star, and a star may further moves around its parent star, until all the daughter galaxies rotate around the center of the galaxy. During a star revolving around its parent star, it unceasingly incorporates the nebula materials near the orbits to become larger and larger. In addition, for a planet, it may gradually move away from its parent star under the impact of moving objects or the radiation of its parent star, even producing progeny galaxies. This means that a star of the $n$th $(n \geq 1)$ generation might produce a sub-galaxy consisting of multiple generations of stars, making its spatial scale become overlong, so that when the sub-galaxy rotates around the star of the $n$th generation, its tail satellite may enter the gravitational field of the star of the (n-1)th generation, and finally be annexed by the later one, greatly increasing the mass and density of the later one, as is shown in Fig. 2.

Moreover, the impact of the sub-galaxy of the $n$th generation star on the (n-1)th generation star will produce tremendous energy, even start thermonuclear reactions, to fuse the satellite with the (n-1)th generation star. Due to the strong gravitation of the center of the (n-1)th generation star, the newly added material shrinks immediately, but conserving the angular momentum of the $(n-1)$ th generation star, so a decrease in the rotational inertia results in an increase in the rotation speed of the $(n-1)$ th generation star, and consequently also an increase in the revolution speed of the $n$th generation star, making the $n$th generation star move away from the (n-1)th generation star, widening the distance between the two generation stars. Similar impacts may occur multiple times, finally allowing the passage of the sub-galaxy of the $n$th generation star. This is the basic principle of the formation and evolution of galactic structure.

Thus, we can see that in some old galaxies which have evolved over a long period of time, there are many high layer stars that have accumulated for a long time and encountered numerous impacts, eventually becoming black holes of great mass and density. Especially, the progenitor at the center of the galaxy has been the earliest and most frequently hit by other celestial objects, and has become a black hole with the largest mass and density. Some daughter stars under or beside the center of the galaxy may also have accumulated for a long time and encountered numerous impacts, eventually becoming massive black holes, but their mass and density are lower than that of their ancestor. Just because the mass of the black hole is very large and the gravitational force is

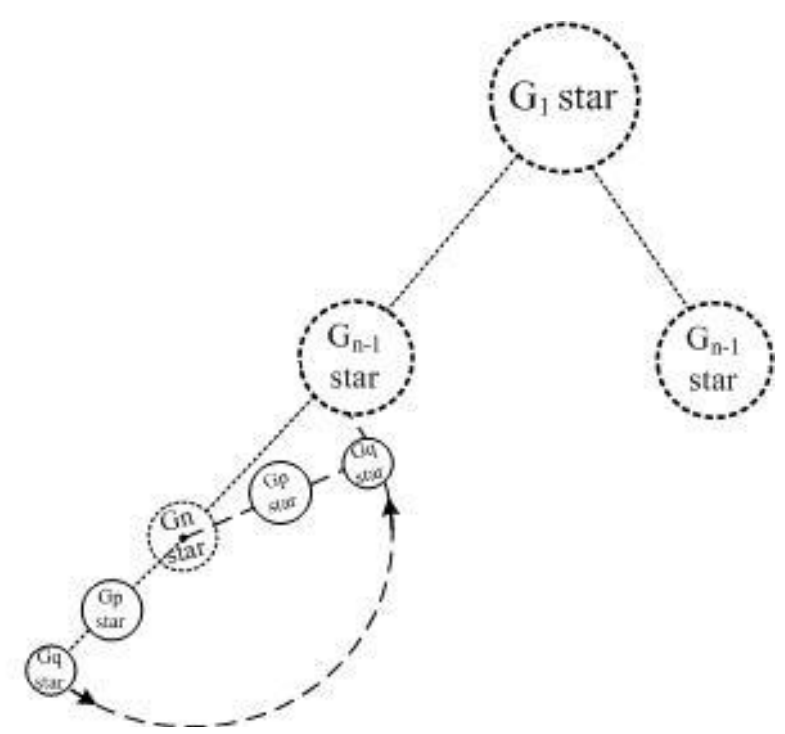

Fig. 2 An overlong sub-galaxy impacting on its parent star makes the mass of the parent star and the distance between the two generation stars increase. 
very strong, any moving body entering the gravitational field of the black hole but having lower speed than light speed cannot escape the trap of the black hole. Also because the mass of the black hole is very large and the gravitational force is very strong, it can attract an extreme thick and dense atmosphere, so that the light emitted by objects smaller than black holes cannot penetrate so thick and dense an atmosphere, just as sunlight cannot penetrate thick clouds. Even if a luminous celestial body enters this area, since lots of its gaseous, liquid and solid materials may be swallowed by the black hole, making the object's resources are insufficient to maintain its luminous effect, causing the phenomenon of "light cannot escape a black hole".

\section{The Formation and Variation of Black Hole Region}

Through a long evolution, an ancient galaxy may contain trillions of stars and have a space scale of thousands to hundreds of thousands of light years. Since so many stars in the galaxy share the resource of nearby cosmic space, and the descendant planets' scope of activity is much larger than that of their parent star, they will win in the competition for resource, gradually growing and finally becoming

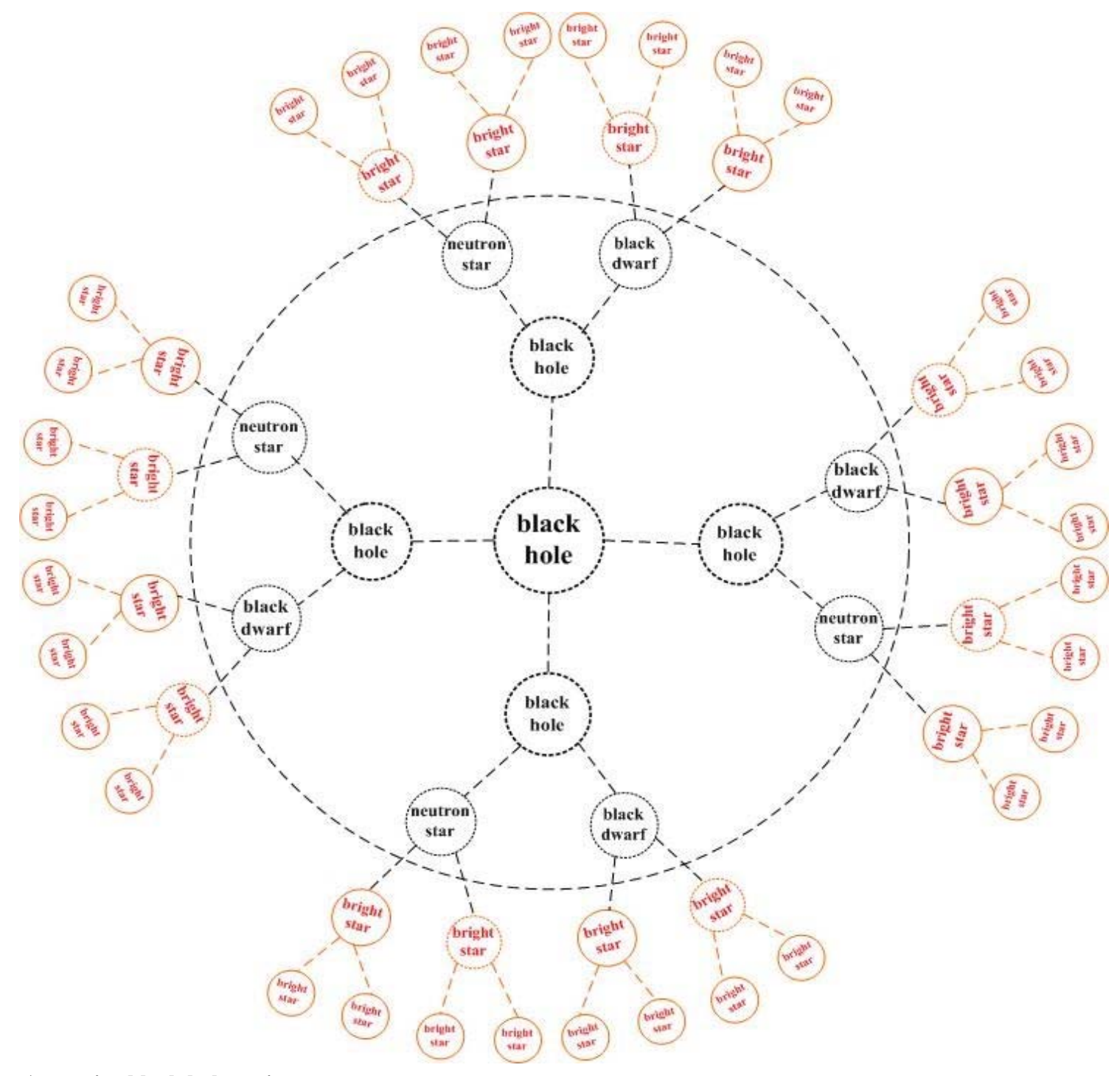

Fig. 3 A massive black hole region. 
glowing stars; while the senior star, due to the layers of the siege of descendant stars, cannot get enough resources to maintain its thermonuclear reaction, finally become a white dwarf. Hence, there are usually at most two bright stars along a single route of a galactic structure. So a galaxy usually has only a few layers of bright stars at the bottom (i.e. the observed periphery), while higher layer stars have become dark. Hence, when the galaxy moves to a place where all luminous stars are located out of a large space, as is shown in Fig. 3, then the large space become a "black hole region". But it is not absolutely empty, instead it may contain hundreds of millions or even billions of white dwarf or neutron star or black dwarf. So the mass of "black hole region" is usually very large. In 2015, a giant double black hole was discovered by Finland scientists, which is virtually a "black hole region". It is the largest black hole ever found in the universe. Its volume is equivalent to the size of the entire Milky Way galaxy, and its mass is 18 billion times that of the sun. But as the bright star around the black hole region moves, the black hole region will also change its shape and size, so the existence of a black hole region is only temporary.

\section{Conclusion}

Since in the past, it is commonly believed that a typical galaxy is composed of many stars embedded in a nebula and the gas, dust and other materials surrounding the stars. Therefore people are blurred about the formation and movement of galaxies, and mistook the black holes hidden in the center of a galaxy as independent celestial objects, making black holes mysterious and unbelievable. Fortunately, the author has studied and discovered the laws of the formation and evolution of satellites, planets and stars, therefore has put forward the scientific theory of galaxy formation and evolution, and revealed the hierarchical structure of galaxy and the existence and characteristics of black holes as the main nodes of galactic structure.

\section{Acknowledgments}

I should thank Professor Ziyuan Ouyang, an academician of the Chinese Academy of Sciences and also an academician of the International Academy of Astronautics, who has provided me with valuable guidance in doing this research.

\section{References}

[1] Gordon Fraser. 2013. The New Physics for the Twenty-First Century. Beijing: Science Press.

[2] Su Yi. 2010. Astronomy for Liberal Arts. Beijing: Science Press.

[3] Hasinger, G., and Team, C. 2003. "Formation and Evolution of Supermassive Black Holes in Galactic Centers: Observational Constraints." AIP Conference Proceedings 666 (1).

[4] Wu, X. B., et al. 2015. "An Ultraluminous Quasar with a Twelve-Billion-Solar-Mass Black Hole at Red Shift 6.30." Nature 518: 512-5 (26 February 2015).

[5] Zhong, C., X. 2016. "The Formation and Evolution of Galaxies and the Expansion of the Universe as well as Dark Matter \& Dark Energy." AASCIT Journal of Phsics, 2016-07-10.

[6] Zhong, C. X. 2016. "The Real Reason for the Earth and Other Planets to Move Away from the Sun as Well as Natural Measures to Mitigate Global Warming." International Journal of Geophysics and Geochemistry, 2016-09-15.

[7] Sackmann, I. J., Boothroyd, A. I., and Kraemer, K. E. 1993. "Our Sun. III. Present and Future." The Astrophysical Journal 418: 457.

[8] Schawinski, K., et al. 2008. "Supernova Shock Breakout from a Red Supergiant." Science 321 (5886): 223-6.

[9] Ramirez, R. M., and Kaltenegger, L. 2016. "Habitable Zones of Post-Main Sequence Stars.” The Astrophysical Journal 823 (1).

[10] Laughlin, G., Bodenheimer, P., and Adams, F. C. 1997. "The End of the Main Sequence." The Astrophysical Journal 482: 420.

[11] Krause, O., et al. 2008. "The Cassiopeia a Supernova Was of Type IIb." Science 320 (5880): 1195-7.

[12] Tauris, T. 2014. "Neutron Star Formation and Evolution-Singles, Binaries and Triples." 40th COSPAR Scientific Assembly, held 2-10 August 2014, in Moscow, Russia.

[13] Haehnelt, M. G., and Kauffmann, G. 2001. "The Formation and Evolution of Supermassive Black Holes and their Host Galaxies." Springer Berlin Heidelberg 2001: 364-74. 